\title{
Highly Luminescent CB[7]-Based Conjugated Polyrotaxanes Embedded into Crystalline Matrices
}

\author{
Talha Erdem, Muazzam Idris, Hilmi Volkan Demir, and Dönüs Tuncel**
}

$\pi$-Conjugated polymers suffer from low quantum yields (QYs) due to chainchain interactions. Furthermore, their emission in solid films is significantly quenched due to aggregation leading further decrease in QY. These are the two main issues of these materials hampering their widespread use in optoelectronic devices. To address these issues, here the backbone of poly $\left(9,9^{\prime}\right.$ bis (6"-(N,N,N-trimethylammonium)hexyl)fluorene-alt-co-thiophenelene) is isolated by threading with cucurbit[7]uril (CB7). Subsequently, the conjugated polyrotaxanes are incorporated into organic crystalline matrices to obtain highly efficient color-converting solids suitable for solid-state lighting. Upon threading the polymer backbone with CB7s, although the QY of the resulting polyrotaxane in solution state increases, the quenching problem in their solid state is not completely tackled. To solve this problem, these conjugated polyrotaxanes are embedded into various crystalline matrices and their remarkably high QYs (>50\%) in the solution are successfully maintained in the solid state. To demonstrate the suitability of these aforementioned materials for solid-state lighting, a proof-of-concept light-emitting diode is constructed by employing their powders as color converters.

they pose significantly lower risks to environment and health compared to other optical materials such as cadmiumbased quantum $\operatorname{dots}^{[10]}$ and lead-based perovskites..$^{[11]}$

Despite the potential of conjugated polymers, a decrease in the fluorescence quantum yield (QY) and a low emission stability in their solid state turn out to be an important bottleneck. These problems mainly arise from the changes in the polymer morphology upon film casting, which affects the inter and intrachain interactions. To decrease these interactions and consequently, achieve higher QYs in solid films, various approaches have been adopted including the utilization of nonradiative energy transfer to light-emitting polymers ${ }^{[12,13]}$ and the incorporation of the polymers into inorganic nanoparticles. ${ }^{[14-16]}$ Alternatively, chemical entities such as dendrones have been attached to the polymer backbone in

\section{Introduction}

Conjugated polymers have emerged as an important class of materials and thus, found applications in the fabrication of various optoelectronic devices. ${ }^{[1-9]}$ Their potential for high efficiency makes them attractive for light-emitting devices while the ability to tune their photophysical features by chemical modifications increases their flexibility. In addition to these,

Dr. T. Erdem, M. Idris, Prof. D. Tuncel

Department of Chemistry and UNAM-National Nanotechnology

Research Center, Institute of Materials Science and Nanotechnology

Bilkent University

Ankara 06800, Turkey

E-mail:dtuncel@fen.bilkent.edu.tr

Prof. H. V. Demir

Departments of Electrical and Electronics Engineering and

Physics, and UNAM-National Nanotechnology Research Center

Institute of Materials Science and Nanotechnology

Bilkent University

Ankara 06800, Turkey

Prof. H. V. Demir

Luminous! Center of Excellence for Semiconductor Lighting and Displays

School of Electrical and Electronic Engineering

School of Physical and Materials Sciences

School of Materials Science and Nanotechnology

Nanyang Technological University

Singapore 639798, Singapore

DOI: 10.1002/mame.201700290 order to weaken the $\pi-\pi$ stacking. ${ }^{[17]}$ Insulation of the conjugated polymer backbones via rotaxanation, which can be considered as threading the polymer chain using a macrocyclic ring, also steps forward as a promising solution. ${ }^{[18-20]}$ In the literature, cyclodextrins and cucurbit $[n]$ urils $(\mathrm{CB}[n])$ have been shown to successfully isolate the polymer backbone leading to enhanced fluorescence QY. ${ }^{[21-26]}$ For instance, our group has recently demonstrated fourfold increase in the fluorescence QY of $\operatorname{poly}\left(9,9^{\prime}\right.$-bis $\left(6^{\prime \prime}-(N, N, N\right.$-trimethylammonium $)$ hexyl)fluorene-alt-co-thiophenelene) accompanied by blue-shifted fluorescence spectrum and prolonged fluorescence lifetime after threading with CB7. ${ }^{[27]}$ Despite the improvements in the QY of polyrotaxane solutions, their solid films still suffered from decreased fluorescence QY. Therefore, a solution to maintain the fluorescence QY is of significant importance for their use in solid-state lighting.

A possible solution to this problem might come from the research on the colloidal quantum dots, which similarly suffered from decreasing QYs in their solid films. Otto et al. ${ }^{[28]}$ showed that the quantum dots in water can be successfully incorporated into crystalline salt matrices. Later, this approach was shown to improve the fluorescence $Q Y^{[29]}$ and emission stability. ${ }^{[30,31]}$ Moreover, this method was also extended to nonionic crystals including sucrose ${ }^{[32]}$ and anthracene ${ }^{[33]}$ and to quantum dots in nonpolar solvents ${ }^{[30,34]}$ and applied in lightemitting diodes (LEDs) ${ }^{[31]}$ and sensors. ${ }^{[35]}$ Recently, our group has evaluated the potential of this technique by incorporating 
the conjugated oligomer nanoparticles, which are essentially wrapped in the form of nanoscale balls of oligomers in sucrose crystals. ${ }^{[36]}$ The QY of the nanoparticles in sucrose powders significantly increased even compared to the nanoparticle solution while the emission stability at elevated temperatures was substantially improved. The increase in the QY caused by the passivation of the nonradiative transitions that are created due to aggregation of polymers while forming nanoparticles.

To solve the emission quenching issue of polymers in the solid films, here we employed both the threading the polymer chains by CB7 forming conjugated polyrotaxanes and incorporating them into a variety of crystalline matrices to form their light-emitting powders. For this purpose, we prepared a polyrotaxane consisting of poly $\left(9,9^{\prime}-\operatorname{bis}\left(6^{\prime \prime}-(N, N, N\right.\right.$ trimethylammonium)hexyl)fluorene-alt-co-thiophenelene) and CB7, and then embedded the polyrotaxane into various hosts including $\mathrm{NaCl}$, sucrose, and trehalose. We observed that the light-emitting powders of the polyrotaxane in the organic matrices exhibit QY up to 52.4\% that is slightly lower than the QY of the polyrotaxane solution (59.1\%) but marginally higher than the QY of their solid films (22.8\%) in the absence of the matrices. Nevertheless, we did not observe any significant enhancement in the QYs when the polymer and polyrotaxane were embedded in the salts. We further showed successfully the applicability of these powders in the solid-state lighting by integrating them with an ultraviolet LED to obtain a green LED.

\section{Results and Discussion}

The syntheses of the polyrotaxane and polymer were carried out as described by Idris et al. ${ }^{[27]}$ In the polyrotaxane, every two repeating units of the polymer backbone contains one CB7 which corresponds to about a 50\% threading efficiency. The molecular structures of the polyrotaxane, polymer, and matrices used in this study are shown in the Figure 1. In order to prepare the samples, the saturated aqueous solutions of $\mathrm{NaCl}$, sucrose, and trehalose were mixed with the aqueous solutions of polymer and polyrotaxane, and the resulting solutions were left for crystallization. Subsequently these crystals were collected and powdered; then, pellets were formed out of these powders for the solid-state photophysical characterization as well to employ them as a color converter on the LED having a peak emission wavelength at $400 \mathrm{~nm}$.
The QYs of the synthesized polymer and polyrotaxane solutions were measured to be $11.0 \%$ and $59.1 \%$ (Figure 2), respectively, supporting the findings in our previous work. ${ }^{[27]}$ We attribute the enhancement in the QY mainly due to the presence of CB7s which decreases the chain-chain interactions by isolating the chains. Interestingly, we did not observe any profound change in the fluorescence spectra of polymer and polyrotaxane solutions (Figure 3), which is not in alignment with our previous findings. ${ }^{[36]}$ We believe that the high concentrations of polymers and polyrotaxanes used in this work may have caused this variation. In parallel with the QY measurements, we observed that the fluorescence lifetime of the polyrotaxane solution is longer than the lifetime of the polymer solution (Figure 4) further supporting our argument on the isolation of polymer backbones by CB7s.

Although a high enhancement was observed in the QY of the polyrotaxane in the solution state, QY decreased to $22.8 \%$ upon forming a film, while that of the polymer solid film remained almost unchanged. The significant shortening of the fluorescence lifetime in the polyrotaxane solid film from 1.1 to $0.7 \mathrm{~ns}$ (Figure 4) and the red-shift in the fluorescence spectrum (Figure 3 ) indicate that during the film formation the aggregation of the polymer chains overwhelms the isolation effect of the CB7s. We also observed similar trends in fluorescence spectrum and lifetime in the solid film of polymer without CB7, which we attribute to the strengthening of the nonradiative transitions. Nevertheless, the QY measurements of these polymers did not reveal any profound variation. This might be because we remained around the detection limits of our spectrometer and indicated also by the large standard deviation in our measurements.

Obviously, the low QY in the solid state significantly limits the applicability of the polymers in the fabrication of optoelectronic devices as color converters. To find a solution to this problem, here we followed the approach of integrating lightemitting materials into crystalline matrices. ${ }^{[37]}$ For this purpose, we embedded our polymers into $\mathrm{NaCl}$, sucrose, and trehalose matrices. We found out that incorporating the polymers into $\mathrm{NaCl}$ or trehalose reduces the QY below the levels of solid film (Figure 2; Figure S1, Supporting Information). Even though polymers in sucrose perform slightly better than other hosts that were tested here, their QY remained to be low as around $10 \%$. This is in contrast with the nanoparticles of conjugated oligomers, which had shown strong improvement in QY when embedded into sucrose host. ${ }^{[36]}$ Different than the conjugated
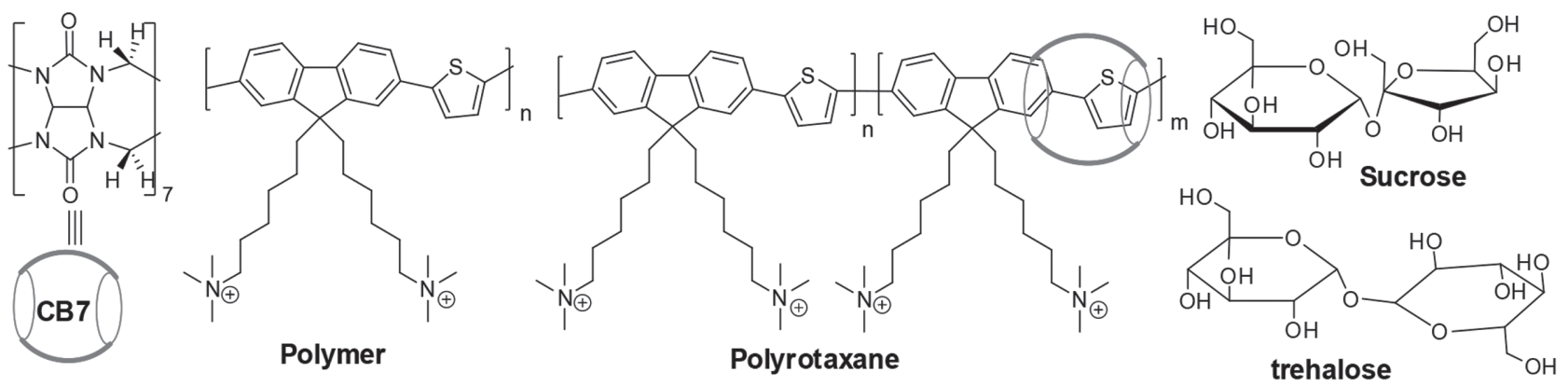

Figure 1. Molecular structures of cucurbit[7]uril (CB7), poly $\left(9,9^{\prime}\right.$-bis $\left(6^{\prime \prime}-(N, N, N\right.$-trimethylammonium) hexyl)fluorene-alt-co-thiophenelene) (polymer) and CB7-threaded poly $\left(9,9^{\prime}\right.$-bis $\left(6^{\prime \prime}\right.$-( $N, N, N$-trimethylammonium $)$ hexyl)fluorene-alt-co-thiophenelene) (polyrotaxane). 


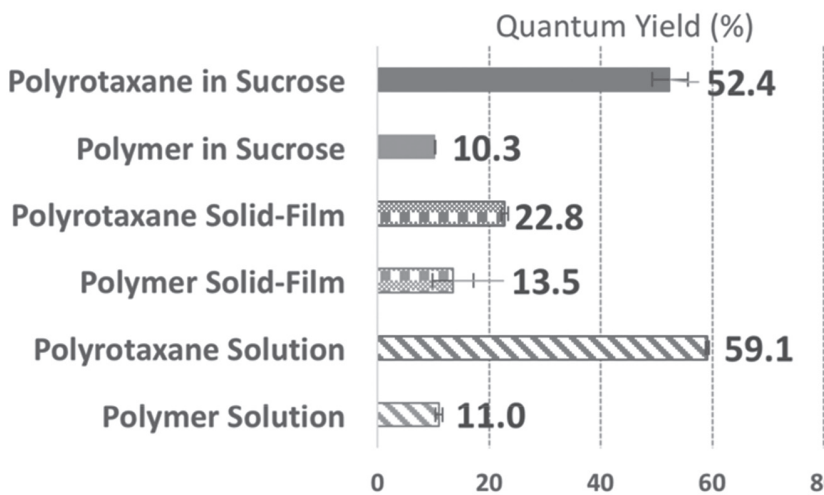

Figure 2. Fluorescence quantum yields of polymer and polyrotaxane solutions, polymer and polyrotaxane solid films, and polymer and polyrotaxane in sucrose host.

oligomer nanoparticles, the polymers in the absence of CB7 encapsulation exhibited a strong red-shift in their fluorescence spectra compared to their solution (Figure 3; Figure S2, Supporting Information) especially at high amounts of loading into the host matrix. This red-shift, however, remained weaker in comparison with the solid films of the polymers. In line with these trends, we measured longer fluorescence lifetimes of the polymers in all the hosts compared to the polymer solid film while they remained shorter in comparison with the fluorescence lifetime of the polymer solution (Figure 4; Figure S3, Supporting Information). All these observations bring us to the conclusion that the molecules of the host medium cannot efficiently passivate the nonradiative transitions of the open polymer chains without CB7-threading as opposed to the case of conjugated oligomer nanoparticles. ${ }^{[36]}$

The polyrotaxanes in crystalline hosts turned out to be more promising for solid-state lighting as shown in Figure 1 and Figure S1 (Supporting Information). Their QYs were measured to be as $52.4 \%$, which is slightly lower than the QY of the polyrotaxane solution but much higher than the QY of the polymer in solid state. Sucrose and trehalose are found to be more suitable hosts for the polyrotaxanes compared to $\mathrm{NaCl}$, which is similar to the case of the nanoparticles of conjugated oligomers. Especially in sucrose, the high QYs of the polyrotaxane solids were accompanied by a negligible red-shift in the fluorescence spectra (Figure 3) and the fluorescence lifetimes converged to the lifetime of the polyrotaxane solution (Figure 4; Figure S3, Supporting Information). These observations further support that the immobilization of the polyrotaxanes within organic hosts, especially in sucrose, helps minimizing the aggregation of the polyrotaxane leading to significantly improved QYs compared to the solid films in the absence of hosts. However, direct observation of the polyrotaxane distribution in a host matrix using electron microscopy has not been possible due to significantly low contrast of the organic molecules in the host matrix. Nevertheless, our previous experiments with the inorganic nanoparticles ${ }^{[32]}$ indicated no significant phase separation. Considering the changes in the optical features of polyrotaxanes described above, we believe that we do not have an important phase separation issue here, as well.

Since the solids of polyrotaxanes in sucrose enable their QYs to be $>50 \%$, this material system might serve as a good color
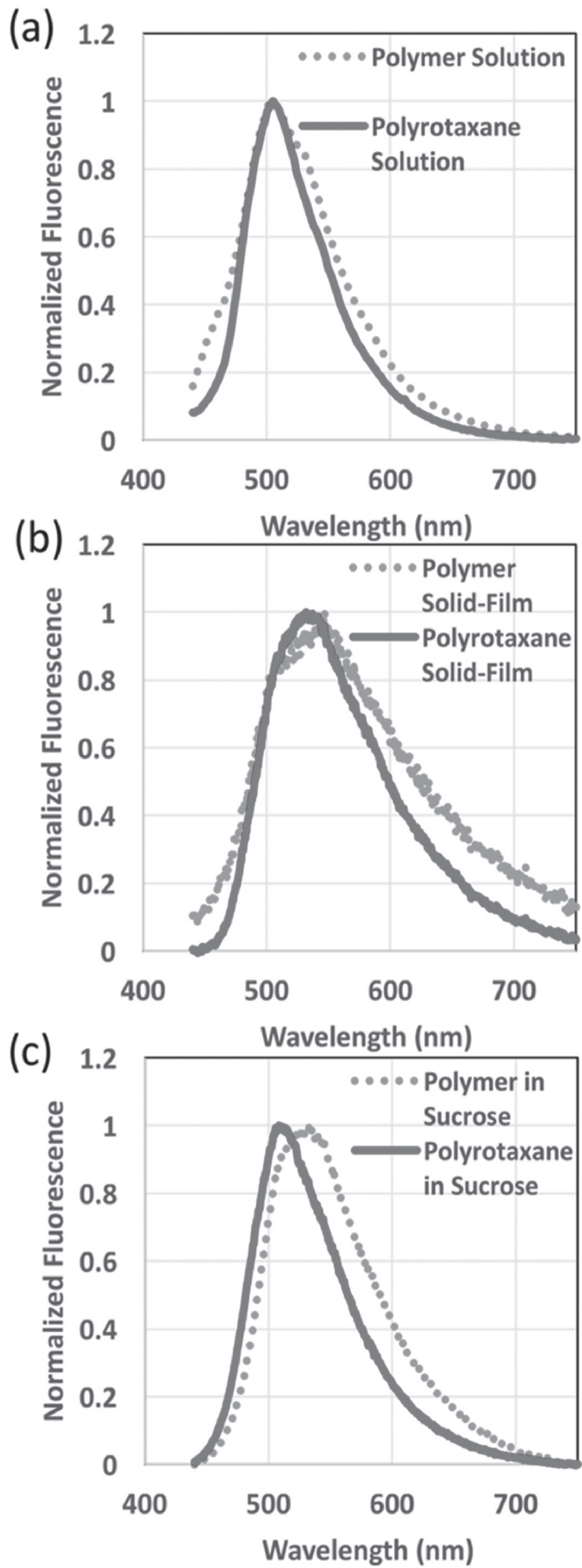

Figure 3. Normalized fluorescence intensities of polymer and polyrotaxane a) solutions and b) solid films in sucrose host.

converter for solid-state lighting applications. With this motivation in mind, we prepared pellets from the powders obtained from the cocrystallization of polyrotaxanes with sucrose using a hydraulic press and the resulting pellets were integrated with a UV-LED emitting at $400 \mathrm{~nm}$. The emission spectra of the LED are presented in Figure 5 along with the color coordinates on the CIE 1931 chromaticity diagram, current dependent luminous flux, and luminous efficiency. Our proof-of-concept 
Lifetime (ns)

Polyrotaxane in Sucrose

Polymer in Sucrose

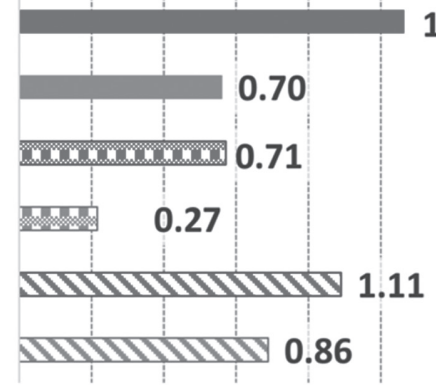

\section{$\begin{array}{lllllll}0 & 0.25 & 0.5 & 0.75 & 1 & 1.25 & 1.5\end{array}$}

Figure 4. Fluorescence lifetimes of polymer and polyrotaxane solutions, polymer and polyrotaxane solid films, and polymer and polyrotaxane in sucrose host.
LED exhibits green emission whose corresponding chromaticity coordinates were $(0.24,0.61)$ at $10 \mathrm{~mA}$ and $(0.26,0.53)$ at $350 \mathrm{~mA}$. The luminous efficiencies took values $>6.5 \mathrm{~lm} / \mathrm{W}_{\text {elect }}$ and the luminous flux became $>6 \mathrm{~lm}$. It is here worth mentioning that the limiting factor of the LED efficiency has been the low power conversion efficiency of the UV-LED rather than the color converting layer.

\section{Conclusion}

In this work, we developed conjugated polyrotaxane-based color converters that can be used on high-power LEDs. For this purpose, the polymer backbone has been isolated by threading with $\mathrm{CB}[7] \mathrm{s}$ to form conjugated polyrotaxanes and then, these polyrotaxanes were incorporated into the crystalline matrices to
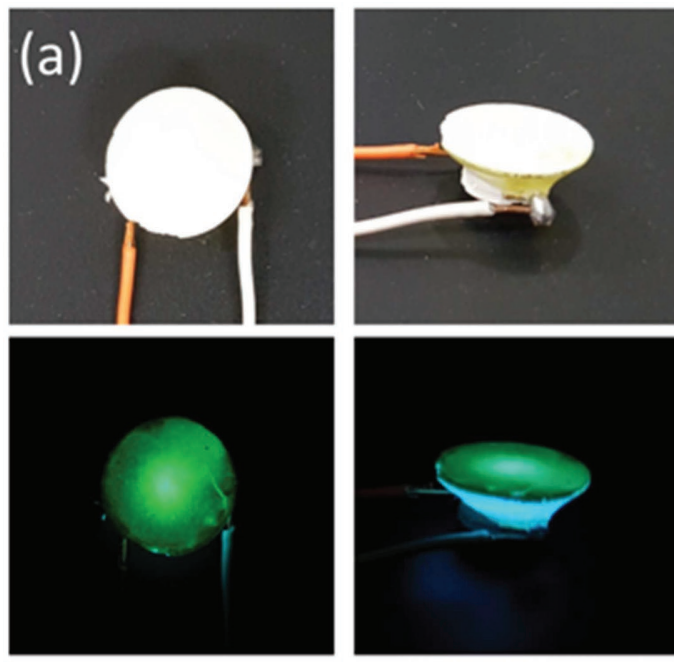

(b)

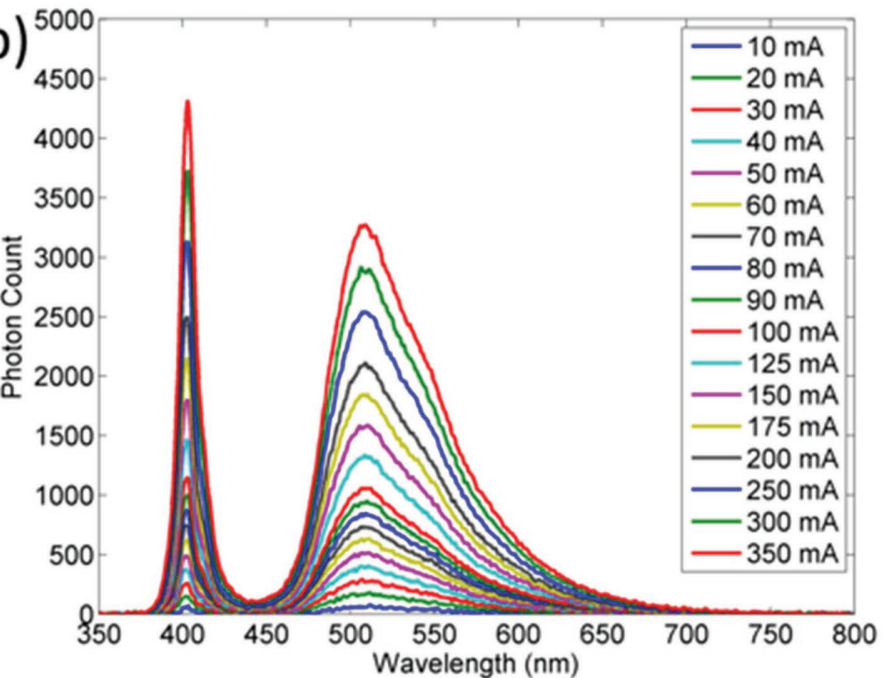

(c)

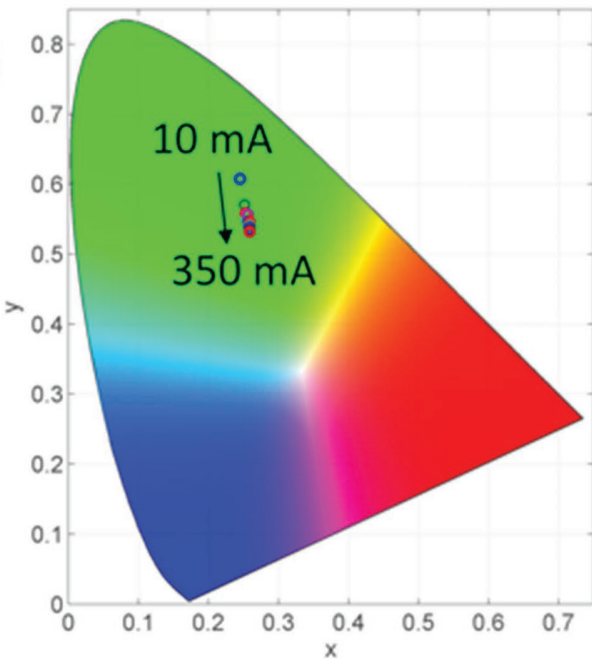

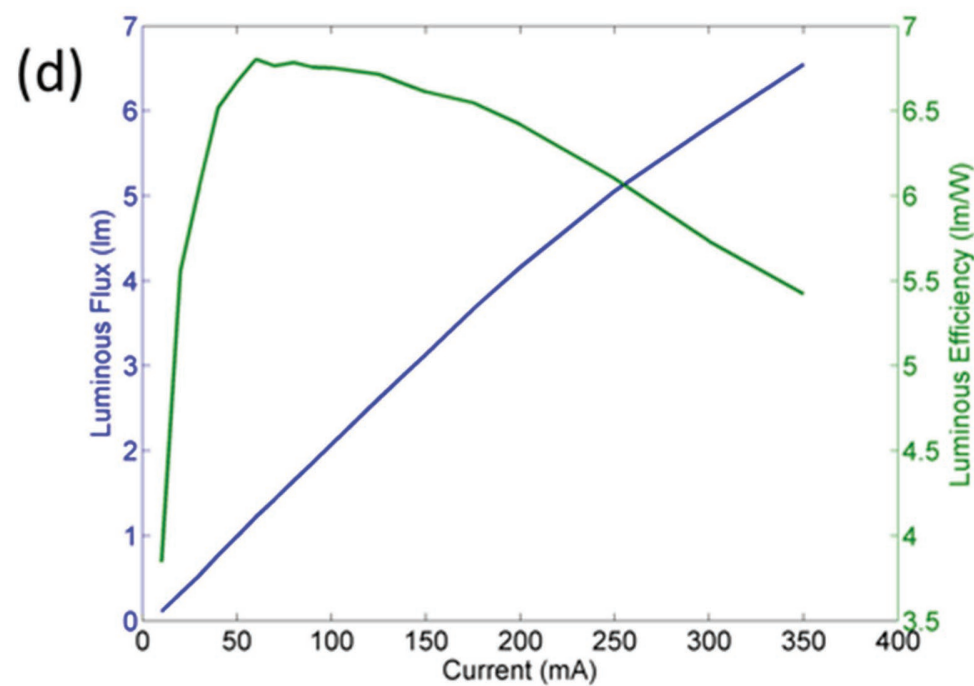

Figure 5. a) Real-color photograph of the polyrotaxane in sucrose host under ambient lighting and during LED operation. b) Emission spectra of the polyrotaxane (in sucrose host) integrated LED at varying current levels. c) CIE 1931 color coordinates of the LED emission between 10 and $350 \mathrm{~mA}$ current flows. d) Luminous flux and luminous efficiency of the LED as a function of the applied current. 
achieve high QYs (>50\%) in the solid state. Subsequently, we employed these materials as color converters on a high-power LED to show their potential for optoelectronic applications.

Remarkably QYs of these light emitting powders of polyrotaxane obtained through the cocrystallization of the polyrotaxane with matrices were measured to be close to the QYs in their solution state and significantly higher than that of the QYs of the polyrotaxane films in the absence of matrices. It turns out to be that the solids of polyrotaxane obtained in organic matrices such as sucrose and trehalose exhibit higher QYs compared to inorganic hosts (e.g., salts).

\section{Experimental Section}

\subsection{Synthesis of Polyrotaxane}

Briefly, 2,7-dibromo-9,9-bis(6- bromohexyl)-9H-fuorene was synthesized following refs. [38] and [39] and treated with trimethylamine solution in tetrahydrofuran to obtain the monomer. The polymer, poly $\left(9,9^{\prime}\right.$-bis $\left(6^{\prime \prime}\right.$. ( $\mathrm{N}, \mathrm{N}, \mathrm{N}$-trimethylammonium)hexyl)fluorene-alt-co-thiophenelene) was obtained by $\mathrm{Pd}(0)$-catalyzed Suzuki-coupling with 2,5-thiophenediboronic ester with 2,7-dibromo-9,9-bis (6- bromohexyl)-9H-fuorene. Polyrotaxane was synthesized by Suzuki-coupling of the CB7-inclusion complex of 2,5-thiophenediboronic ester with 2,7-dibromo-9,9-bis (6- bromohexyl)$9 \mathrm{H}$-fuorene in the presence of $\mathrm{Pd}(0)$-catalyst (Scheme S1, Supporting Information). For every two repeating units in the polymer backbone, one thiophene is encapsulated by CB7 which corresponds to about a $50 \%$ threading efficiency.

\subsection{Incorporation of the Polymers and Polyrotaxanes into Crystalline Matrices and the Fabrication of the LED}

The concentration of the synthesized polymers without $\mathrm{CB}[7]$ and polyrotaxanes of $\mathrm{CB}[7]$ in water was set to $0.2 \mathrm{mg} \mathrm{mL}$. All optical characterizations of the solutions were carried out at these concentrations. The solid films were prepared by drop-casting $50 \mu \mathrm{L}$ of these solutions on a glass substrate. Subsequently, 1,2 , and $3 \mathrm{~mL}$ of the polyrotaxane or polymer solution were mixed with $2 \mathrm{~mL}$ of saturated $\mathrm{NaCl}$ solution, sucrose solution $\left(520 \mathrm{~g} \mathrm{~L}^{-1}\right)$, or trehalose solution $\left(800 \mathrm{~g} \mathrm{~L}^{-1}\right)$. For the mixtures having volumes less than $5 \mathrm{~mL}$, $\mathrm{ddH}_{2} \mathrm{O}$ was added to achieve a total volume of $5 \mathrm{~mL}$. Corresponding concentrations of the polymers and polyrotaxane in the crystallization solution become 4,8 , and $12 \% \mathrm{w} / \mathrm{v}$, respectively. These mixtures were left to crystallize under ambient temperature and pressure on a vibration absorbing pad. The incorporation levels of the added molecules were tested by redissolving the powders in water, measuring the absorbance levels, and comparing with the polyrotaxane solution indicated almost complete incorporation, which has been the case for our previous works as well. ${ }^{[36]}$ Obtained crystals were then powdered using a mortar. A pellet of the polyrotaxane powders ( $12 \% \mathrm{w} / \mathrm{v}$ in sucrose) was prepared using a hydraulic press at a pressure of 5400 psi applied for 5 min. This pellet was then integrated with an ultraviolet LED emitting at $400 \mathrm{~nm}$ by placing first $\approx 250 \mu \mathrm{L}$ of transparent epoxy (Bison 2-component epoxy) on the pellet and pasting the LED to the pellet.

\subsection{Optical Characterizations}

The fluorescence measurements of the polymer and polyrotaxane solutions were carried out using a Cary Eclipse Fluorescence Spectrometer, the absorbance spectra of the solutions were taken using Cary 100 UV-vis spectrometer, quantum yield measurements were carried out as described in ref. [30], the absorption spectrum of the powders was measured using an Ocean Optics integrating sphere equipped with an Ocean Optics Maya 2000 spectrometer and a Xenon lamp (Spectral Products). The emission spectra, optical power, and luminance of the LED were measured using the same integrating sphere and spectrometer. Time-resolved fluorescence decays of the polymer and polyrotaxane solutions, their solid powders in crystalline matrices, and their solid films were measured using a Picoquant Fluotime 200 time correlated single photon counting system. The fluorescence lifetimes were measured by fitting the experimental decays using multiexponential functions and calculating amplitude-averaged lifetimes. ${ }^{[40]}$ We measured the fluorescence quantum yield of the polymer and polyrotaxane solutions in water using the method described in refs. [30] and [41].

\section{Supporting Information}

Supporting Information is available from the Wiley Online Library or from the author.

\section{Acknowledgements}

D.T. acknowledges TUBITAK-TBAG 112T058, KBAG 114Z195, and COST Action CM1005 (Supramolecular Chemistry in Water). H.V.D. gratefully acknowledges support from TÜBA.

\section{Conflict of Interest}

The authors declare no conflict of interest.

\section{Keywords}

color converters, conjugated polymers, light-emitting materials macrocrystals, polyrotaxanes

Received: June 14, 2017

Revised: August 2, 2017

Published online: September 12, 2017

[1] V. Podzorov, Nat. Mater. 2013, 12, 947

[2] H.-F. Meng, in Polymer Electronics (Ed: H.-F. Meng), Stanford Publishing Pte. Ltd., Singapore 2013.

[3] A. Facchetti, Chem. Mater. 2011, 23, 733.

[4] L. Feng, C. Zhu, H. Yuan, L. Liu, F. Lv, S. Wang, Chem. Soc. Rev. 2013, 42, 6620.

[5] A. C. Grimsdale, K. L. Chan, R. E. Martin, P. G. Jokisz, A. B. Holmes, Chem. Rev. 2009, 109, 897.

[6] S. E. Root, S. Savagatrup, A. D. Printz, D. Rodriquez, D. J. Lipomi, Chem. Rev. 2017117, 6467.

[7] I. Fischer, A. P. H. J. Schenning, in Organic Electronics: Emerging Concepts and Technologies (Eds: C. Cicoira, F. Santato), Wiley-VCH Verlag GmbH \& Co. KGaA, Germany 2013, pp. 1-25.

[8] F. Kong, X. L. Wu, G. S. Huang, R. K. Yuan, P. K. Chu, Thin Solid Films 2008, 516, 6287.

[9] H. Keita, B. Guzelturk, J. Pennakalathil, T. Erdem, H. V. Demir D. Tuncel, J. Mater. Chem. C 2015, 3, 10277.

[10] L. Y. T. Chou, W. C. W. Chan, Nat. Nano 2012, 7, 416

[11] A. Babayigit, A. Ethirajan, M. Muller, B. Conings, Nat. Mater. 2016 $15,247$.

[12] I. O. Ozel, T. Ozel, H. V. Demir, D. Tuncel, Opt. Express 2010, 18, 670.

[13] T. Erdem, V. Ibrahimova, D. Jeon, I. Lee, D. Tuncel, H. V. Demir, J. Phys. Chem. C 2013, 117, 18613 
[14] B. Zhu, Y. Han, M. Sun, Z. Bo, Macromolecules 2007, 40, 4494.

[15] T. Heek, C. Fasting, C. Rest, X. Zhang, F. Wurthner, R. Haag, Chem. Commun. 2010, 46, 1884

[16] S. Kirmayer, E. Dovgolevsky, M. Kalina, E. Lakin, S. Cadars, J. D. Epping, A. Fernández-Arteaga, C. Rodríguez-Abreu, B. F. Chmelka, G. L. Frey, Chem. Mater. 2008, 20, 3745.

[17] K. Liu, Y. Yao, Y. Kang, Y. Liu, Y. Han, Y. Wang, Z. Li, X. Zhang, Sci. Rep. 2013, 3, 2372

[18] G. Wenz, B.-H. Han, A. Müller, Chem. Rev. 2006, 106, 782.

[19] M. J. Frampton, H. L. Anderson, Angew. Chem., Int. Ed. 2007, 46, 1028.

[20] J. Terao, Polym. Chem. 2011, 2, 2444.

[21] R. Eelkema, K. Maeda, B. Odell, H. L. Anderson, J. Am. Chem. Soc. 2007, 129, 12384.

[22] N. Willis-Fox, C. Belger, J. F. Fennell, R. C. Evans, T. M. Swager, Chem. Mater. 2016, 28, 2685.

[23] D. Tuncel, M. Artar, S. B. Hanay, J. Polym. Sci., Part A: Polym. Chem. 2010, 48, 4894.

[24] H. Bai, H. Chen, R. Hu, M. Li, F. Lv, L. Liu, S. Wang, ACS Appl. Mater. Interfaces 2016, 8, 31550.

[25] H. Bai, H. Yuan, C. Nie, B. Wang, F. Lv, L. Liu, S. Wang, Angew. Chem., Int. Ed. 2015, 54, 13208.

[26] H. Bai, H. Zhang, R. Hu, H. Chen, F. Lv, L. Liu, S. Wang, Langmuir 2017, 33, 1116

[27] M. Idris, M. Bazzar, B. Guzelturk, H. V. Demir, D. Tuncel, RSC Adv. 2016, 6, 98109.

[28] T. Otto, M. Müller, P. Mundra, V. Lesnyak, H. V. Demir, N. Gaponik, A. Eychmüller, Nano Lett. 2012, 12, 5348.
[29] M. Müller, M. Kaiser, G. M. Stachowski, U. Resch-Genger, N. Gaponik, A. Eychmüller, Chem. Mater. 2014, 26, 3231.

[30] T. Erdem, Z. Soran-Erdem, V. K. Sharma, Y. Kelestemur, M. Adam, N. Gaponik, H. V. Demir, Nanoscale 2015, 7, 17611.

[31] M. Adam, T. Erdem, G. M. Stachowski, Z. Soran-Erdem, J. F. L. Lox, C. Bauer, J. Poppe, H. V. Demir, N. Gaponik, A. Eychmüller, ACS Appl. Mater. Interfaces 2015, 7, 23364.

[32] T. Erdem, Z. Soran-Erdem, P. L. Hernandez-Martinez, V. K. Sharma, H. Akcali, I. Akcali, N. Gaponik, A. Eychmüller, H. V. Demir, Nano Res. 8, 860.

[33] Z. Soran-Erdem, T. Erdem, P. L. Hernandez-Martinez, M. Z. Akgul, N. Gaponik, H. V. Demir, J. Phys. Chem. Lett. 2015, 6, 1767.

[34] M. Adam, Z. Wang, A. Dubavik, G. M. Stachowski, C. Meerbach, Z. Soran-Erdem, C. Rengers, H. V. Demir, N. Gaponik, A. Eychmüller, Adv. Funct. Mater. 2015, 25, 2638.

[35] S. Kalytchuk, O. Zhovtiuk, S. V. Kershaw, R. Zbořil, A. L. Rogach, Small 2016, 12, 466.

[36] Z. Soran-Erdem, T. Erdem, K. Gungor, J. Pennakalathil, D. Tuncel, H. V. Demir, ACS Nano 2016, 10, 5333.

[37] M. Adam, N. Gaponik, A. Eychmüller, T. Erdem, Z. Soran-Erdem, H. V. Demir, J. Phys. Chem. Lett. 2016, 7, 4117

[38] I. O. Huyal, U. Koldemir, T. Ozel, H. V. Demir, D. Tuncel, J. Mater. Chem. 2008, 18, 3568.

[39] V. Ibrahimova, S. Ekiz, Ö. Gezici, D. Tuncel, Polym. Chem. 2011, 2, 2818

[40] J. C. de Mello, H. F. Wittmann, R. H. Friend, Adv. Mater. 1997, 9, 230.

[41] J. R. Lakowicz, Principles of Fluorescence Spectroscopy, Springer, US 2009 